\title{
Analysis of the mechanism by which nerve growth factor promotes callus formation in mice with tibial fracture
}

\author{
XI-GUANG SANG ${ }^{1}$, ZHI-YONG WANG ${ }^{1}$, LIN CHENG $^{1}$, \\ YAN-HONG LIU ${ }^{2}$, YONG-GANG LI ${ }^{1}$, TAO QIN ${ }^{1}$ and KAI DI ${ }^{1}$ \\ Departments of ${ }^{1}$ Emergency Surgery and ${ }^{2}$ Laboratory Medicine, Qilu Hospital \\ of Shandong University, Jinan, Shandong 250012, P.R. China
}

Received March 2, 2016; Accepted October 26, 2016

DOI: $10.3892 /$ etm.2017.4108

\begin{abstract}
The aim of the present study was to analyze the mechanism by which nerve growth factor (NGF) promotes callus formation in mice with tibial fracture. NGF transgenic homozygotic mice and NGF wild homozygotic mice were selected to construct non-stabilized fracture model of tibia. The mice were sacrificed on days 7,14 and 21, respectively, and each group had a sample with 8 mice at each point in time. X-ray radiography and safranin fast green were used to observe fracture healing and in situ hybridization was used to examine the NGF mRNA expression of tibia at each phase of fracture healing. Tartrate-resistant acid phosphatase (TRAP) staining of callus tissue and the expression level of TRAP mRNA were combined to observe osteoclast formation. COL2Al, a chondrocyte differentiation-related gene in callus, and the mRNA level of SOX9 were combined to observe chondrocyte differentiation. It was found that under $\mathrm{X}$-ray radiography, the fracture of NGF transgenic homozygotic mice healed in advance $(\mathrm{P}<0.05)$. Cartilage and bone tissue were identified by safranin and fast green staining. The residual cartilage on the callus tissue of NGF transgenic homozygotic mice had decreased significantly $(\mathrm{P}<0.05)$. The NGF mRNA expression level in each phase of callus formation of NGF transgenic homozygotic mice was significantly higher than that of the wild group $(\mathrm{P}<0.05)$. The number of positive cells in NGF-TRAP staining at each time point after fracture and the NGF mRNA expression level was markedly higher than that of the wild group, and the expression levels of COL2A1 and SOX9 mRNA were distinctively higher than that of the wild group. In conclusion, NGF potentially improves the healing of tibial fracture by osteoclast formation. Additionally, an increase in the number of osteoblasts in the NGF transgenic
\end{abstract}

Correspondence to: Dr Xi-Guang Sang, Department of Emergency Surgery, Qilu Hospital of Shandong University, 107 Wen Hua Xi Road, Jinan, Shandong 250012, P.R. China

E-mail: xi_guangsang1@163.com

Key words: nerve growth factor, callus, tibial fracture, transgenic mice, multiple trauma homozygotic mice compared with the wild-type mice may be achieved by cartilage differentiation due to NGF increasing the COL2A1 and SOX9 mRNA expression levels.

\section{Introduction}

Nerve growth factor (NGF) is widely applied in the clinic and plays a significant role in neuronal survival support, peripheral nerve growth and nutrition adaptation, nerve regeneration and fracture repair (1). Examination of fracture and the regional callus tissue identified a high expression of NGF and its receptor (2). Local injection of exogenous NGF facilitated the complete healing of the fracture and reduced the formation of heterotopic ossification (3). Therefore, NGF is a potential action target for the prognosis of clinical fracture improvement. However, its precise mechanism is not yet fully understood since NGF action on bone tissue is multifaceted, multi-level and overlapping (4).

Fracture healing process may require a variety of cytokines, such as transforming growth factor- $\beta$, insulin-like growth factor, bone morphogenetic protein and vascular endothelial growth factor (5). However, whether NGF plays a key role in the fracture healing process, a continuous role in the process of callus formation, or whether there is any association with osteoclast formation and cartilage differentiation remains to be determined. In addition, exogenous NGF injection was applied previously to observe its effect on fracture healing, thus different conclusions may be drawn due to the differences in appropriate dose and the injection site (6).

Non-interventional observation on stabilized transgenic mice was used in the present study to identify the specific mechanism involved in fracture healing of endogenous NGF.

\section{Materials and methods}

Animals. A total of 48 NGF wild homozygotic mice $(\mathrm{n}=24)$ and NGF transgenic homozygotic mice $(\mathrm{n}=24)$ were provided by JOINN Laboratories Inc. (Suzhou, China). Quantitative polymerase chain reaction (qPCR) amplification technology was utilized for gene segment identification in agarose gel electrophoresis (AGE). The mice were specific-pathogen-free male mice aged 6 weeks and weighing $180 \pm 20 \mathrm{~g}$. The animals were kept in cages with an ambient temperature of $20-25^{\circ} \mathrm{C}$ 
and humidity $60 \%$, were fed a normal diet and had access to water ad libitum, and were subjected to a 12-h dark/light cycle.

Main reagents and equipment. The reagents used for the study were tartrate-resistant acid phosphatase (TRAP) staining kit (Sigma, St. Louis, MO, USA), phosphate-buffered saline (PBS) (Gibco, Grand Island, NY, USA), TRIzol (Invitrogen Life Technologies, Carlsbad, CA, USA), RNA reverse transcription system (Takara Bio, Inc., Otsu, Japan), X-ray film (Kodar, Rochester, NY, USA), safranin O and fast green (Sinopharm Chemical Reagent Co., Ltd., Shanghai, China), and digoxin RNA labeling kit (Roche Diagnostics, Indianapolis, IN, USA).

An animal trace X-ray machine (Faxitron X-ray Corp., Wheeling, IL, USA), MX3000P qPCR System (Stratagene, La Jolla, CA, USA), gel imaging device (Bio-Rad, Berkeley, CA, USA), ultraviolet (UV) spectrophotometer (Eppendorf AG, Hamburg, Germany), RM2135 microtome (Leica, Mannheim, Germany), Spot built-in digital color camera (Diagnostic Instruments Inc., Sterling Heights, MI, USA), and $-70^{\circ} \mathrm{C}$ cryogenic freezer (Sanyo, Tokyo, Japan) constituted the equipment used.

Fracture models. Non-stabilized tibial fracture model of mice was established as described in a previous study by Kim et al (7). Intraperitoneal anesthetic of 2.5\% Avertin $0.012-0.018 \mathrm{ml} / \mathrm{g}$ was injected into the mice. The skin at middle tibia of its right lower limb was excised longitudinally, with a cut of approximately $0.5 \mathrm{~cm}$ in length. The tibia was transected with scissors following removal of the muscles at middle tibia, and then the incision was sutured layer by layer. The animals were sacrificed by cervical dislocation after 7 , 14 and 21 days of the fracture. The animal skin was cut on the right limb to expose knee joint and tibia. The femur was dissected at $0.5 \mathrm{~cm}$ above the knee joint and the tibia was cut at $0.5 \mathrm{~cm}$ below the fracture. The model was then rinsed with PBS, fixed, decalcified, dewaxed, embedded and sectioned.

Fracture healing situations. X-ray radiography and safranin-fast green staining were applied to observe fracture healing. Each group had 8 mice samples at the 7th, 14th and 21st day, respectively. Giotto FFDM system (Broomfield, CO, USA) was applied in the X-ray photography of the fracture. The main procedures of safranin-fast green staining included, staining with hematoxylin for 20-30 sec, rinsing in running water for $8 \mathrm{~min}, 1 \%$ hydrochloric acid-ethanol color separation for $40 \mathrm{sec}$, rinsing in running water for $5 \mathrm{~min}, 2 \%$ fast green staining for $3 \mathrm{~min}$, fast rinse in $1 \%$ acetic acid, rinsing in clean water, $0.1 \%$ safranin $\mathrm{O}$ staining for 2.5-3 min, rinsing in clean water, and $10 \mathrm{sec}$ in $95 \%$ alcohol for $2 \mathrm{~min}$ in $100 \%$ alcohol. Mounting in neutral balsam was initiated after xylene became transparent. The results showed the cartilage as red stained and the bone tissue as green stained.

In situ hybridization to examine the expression of $N G F m R N A$ in tibia. A Digoxin RNA labeling kit was used to prepare the RNA probe which was complementary to Digoxin labeling in accordance with the manufacturer's instructions. The main steps of in situ hybridization included sequential treatment with $0.2 \mathrm{~mol} / 1 \mathrm{HCl}$ at room temperature for $10 \mathrm{~min}, 1 \mathrm{X}$ PBST solution for $5 \mathrm{~min} \times 2$ times, $20 \mu \mathrm{g} / \mathrm{ml}$ proteinase $\mathrm{K}$ solution
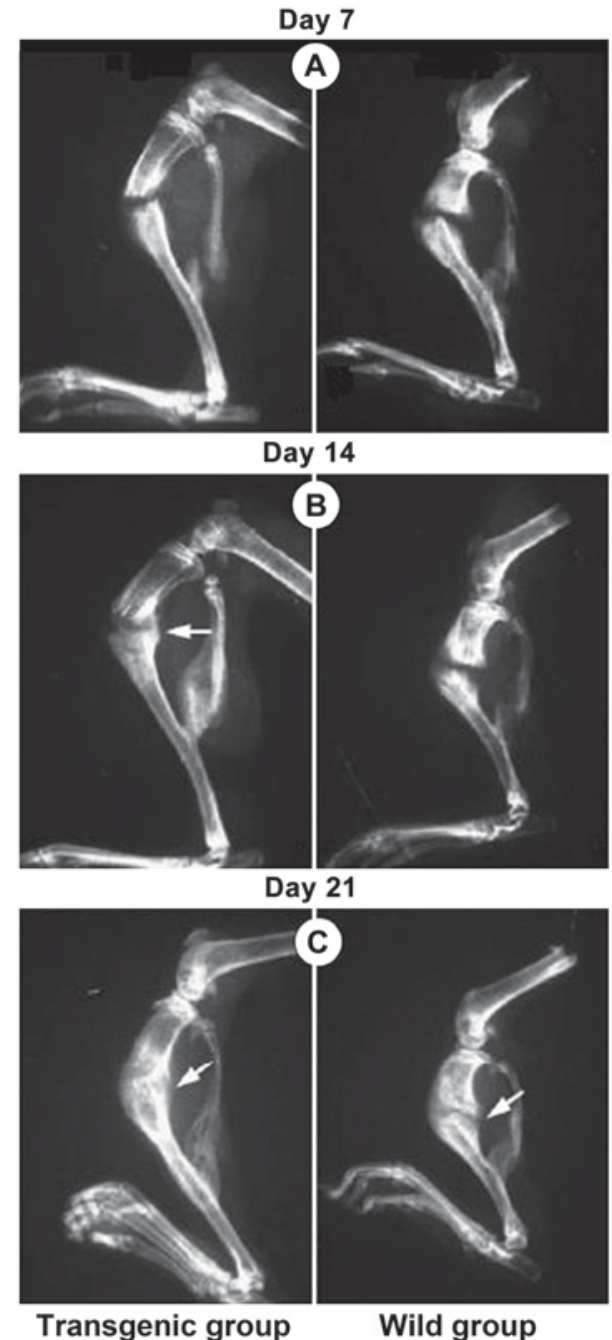

Figure 1. X-ray radiography. (A) Fracture lines were obviously shown in both groups at the 7th day; (B) osseous bridge has obviously appeared in transgenic group at the 14th day of fracture, while it was small in the wild group; (C) osseous union has appeared in the transgenic group at the 21 st day of fracture and the fracture was healed. Delayed healing was evident in the wild group.

digestion at $37^{\circ} \mathrm{C}$ for $15 \mathrm{~min}$, and washing in PBST solution for 5 min $x 2$ times. The probe was fixed in $4 \%$ paraformaldehyde for $10 \mathrm{~min}, 10 \mathrm{~min}$ for $0.1 \mathrm{~mol} / \mathrm{l}$ acetic anhydride/triethanolamine, and finally $50^{\circ} \mathrm{C}$ pre-hybridization for $1 \mathrm{~h}$. The RNA probe was denatured after $85^{\circ} \mathrm{C}$, pre-hybridized, dropped and hybridized at $50^{\circ} \mathrm{C}$ for overnight. The hybridisation buffer sample without the probe was considered the negative control. The film was washed, and confined in confining liquid at room temperature for $1 \mathrm{~h}$. Primary rabbit polyclonal anti-digoxin antibody (dilution: 1/1000; Abcam, Cambridge, MA, USA; catalog no.: ab30512) was used for incubation at $37^{\circ} \mathrm{C}$ for $2 \mathrm{~h}$, and the sample with TBST only was used as the negative control. After rinsing, the NBT/BCIP liquid was kept in the dark to develop color for $4 \mathrm{~h}$, and then counterstained with methyl green, dehydrated and mounted. It was found that positive cells of in situ hybridization were cytoplasm or nucleus, which were stained in purplish red or black blue.

Callus tissue TRAP staining. Osteoclast formation was observed through callus tissue TRAP staining and the TRAP 
Day 7

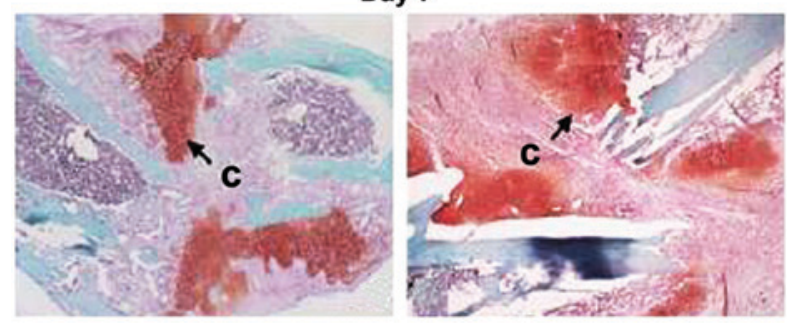

Day 14

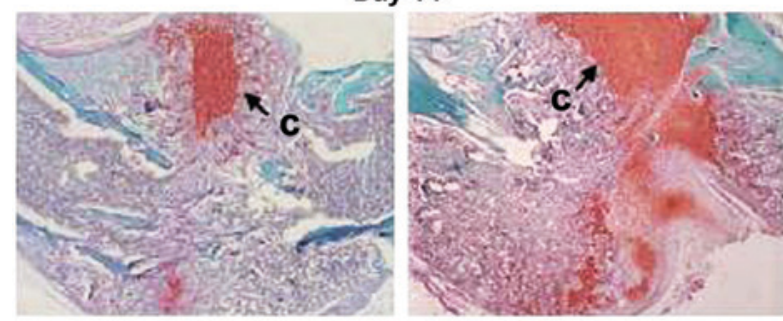

Day 21

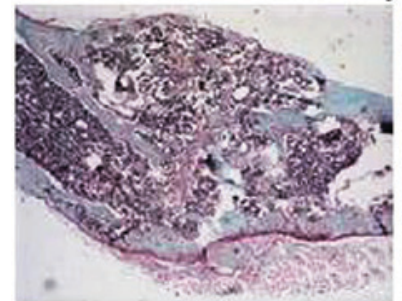

Transgenic group

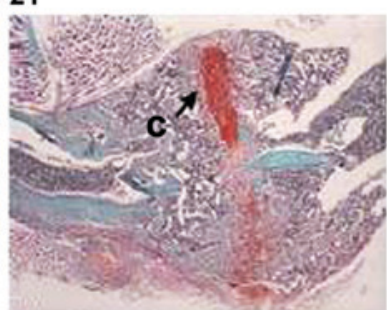

Wild group
Figure 2. Safranin and fast green staining (magnification, $x 40$ ). The cartilage formation of the two groups had no significant differences 7 days after the fracture; at the 14th day, the transgenic group entered bony callus period with cartilage replaced by a large number of new woven bones and significant reduction of cartilage tissues; at the 21st day, cartilage disappeared and marrow cavity began to form in the transgenic group, while there were still many cartilage tissues in the wild group and marrow cavity was not yet evident.

mRNA expression levels. TRAP staining kit was applied in strict accordance with operating instructions.

Quantitative fluorescent PCR method used to detect chondrocyte differentiation-related genes (COL2A1 and SOX9). According to the TRIzol reagent protocol, the one step method was applied to extract total RNA of tissues. AGE was used to examine its integrity, and the total RNA concentration and purity were measured using a UV lamp and UV spectrophotometer. The primers used in the study were: COL2A1 (5'-CTG GTGGAGCAGCAAGAGCAA-3' and 5'-CAGTGGACAGTA GACGGAGGAAAG-3'), SOX9 (5'-GGGCTCTACTCCACC TTCACT-3' and 5'-AAGATCAGCTCGGTCACCATA-3'), $\beta$-actin cyclophillin A (5'-CGAGCTCTGAGCACTGGAGA-3' and 5'-TGGCGTGTAAAGTCACCACC-3'). The amplification conditions used were: $95^{\circ} \mathrm{C}$ for $30 \mathrm{sec}, 95^{\circ} \mathrm{C}$ for $5 \mathrm{sec}, 57^{\circ} \mathrm{C}$ for $20 \mathrm{sec}, 72^{\circ} \mathrm{C}$ for $15 \mathrm{sec}, 40$ cycles (amplification); $95^{\circ} \mathrm{C}$ for $30 \mathrm{sec}, 57^{\circ} \mathrm{C}$ for $30 \mathrm{sec}, 95^{\circ} \mathrm{C}$ for $30 \mathrm{sec}$ (dissociation curve). The value of c (q) and the relative content of the target gene were provided using MX3000P qPCR instrument software (Stratagene).

Statistical analysis. SPSS 19.0 statistical software (SPSS, Inc., Chicago, IL, USA) was applied for data entry and analysis. Measurement data were presented as mean \pm standard
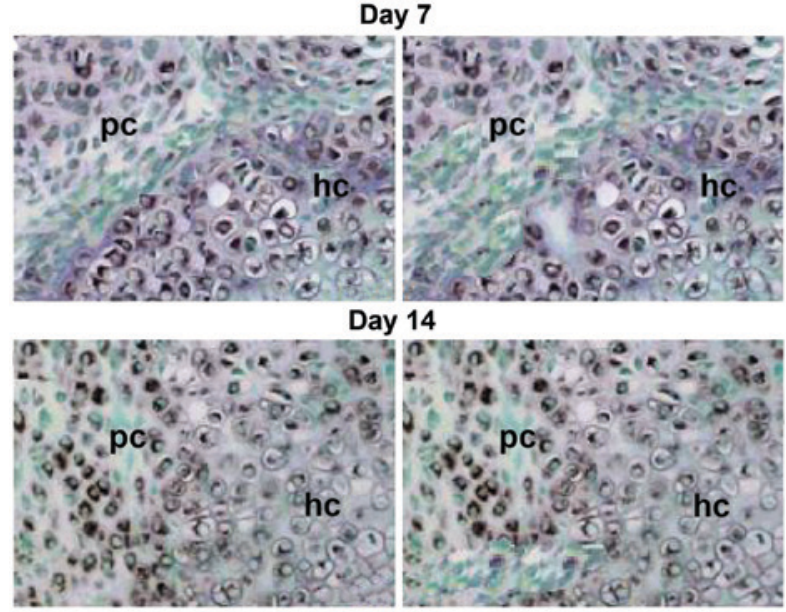

Day 21

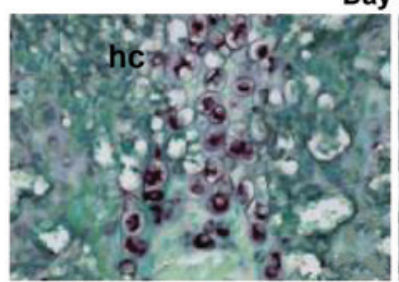

Transgenic group

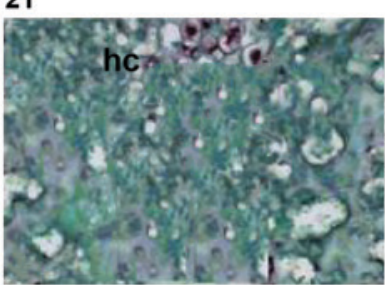

Wild group
Figure 3. In situ hybridization (magnification, x400). At 7 days after the fracture the cartilage callus formation phase occurred, and NGF mRNA expression was detected in pc and hc; 14 days after the fracture, the bony callus formation phase occurred and NGF mRNA expression was detected in pc and hc albeit its amount was reduced; 21 days after the fracture, cartilage tissues disappeared in general. The NGF mRNA expression level was only detected in hc, with a decreased expression than previously. pc, proliferating chondrocyte; hc, hypertrophic chondrocyte.

deviation. Group comparisons were made using single-factor ANOVA. Countable data were expressed as a percentage (\%) and group comparisons were tested using the $\chi^{2}$ test. $\mathrm{P}<0.05$ was considered to indicate a statistically significant difference.

\section{Results}

$X$-ray radiography and safranin fast green for fracture healing observation. Mice fracture models in each group were successfully established. Under X-ray radiography observation, the fracture of NGF transgenic homozygotic mice healed in advance (Fig. 1). Cartilage and bone tissue were expressed by safranin and fast green staining. The residual cartilage on callus of NGF transgenic homozygotic mice had decreased significantly (Fig. 2).

NGF mRNA expression in each callus formation phase. NGF mRNA expression levels in each callus formation phase in NGF transgenic homozygotic mice was significantly higher than that of the wild group (Fig. 3).

TRAP mRNA expression levels. The number of positive cells in NGF-TRAP staining at each time point after the fracture and the expression level of NGF mRNA were significantly higher than that of the wild group (Fig. 4).

COL2A1 and SOX9 mRNA expression levels. The COL2A1 and SOX9 mRNA expression levels of NGF-TRAP stained 
A

Expression relative to Cyclophillin A

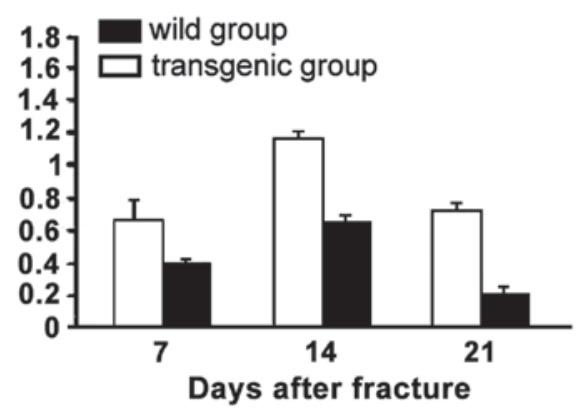

B

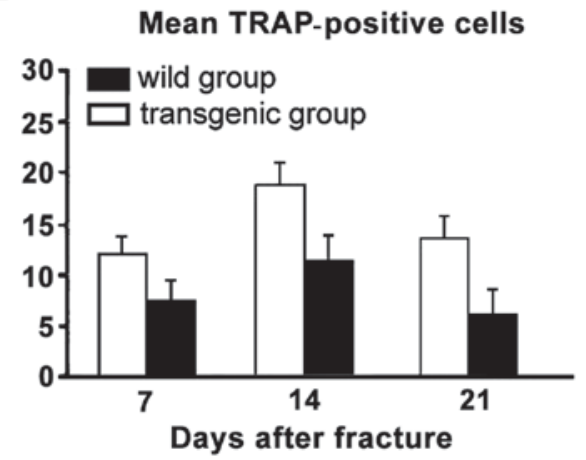

Figure 4. (A and B) Tartrate-resistant acid phosphatase (TRAP) staining.

A

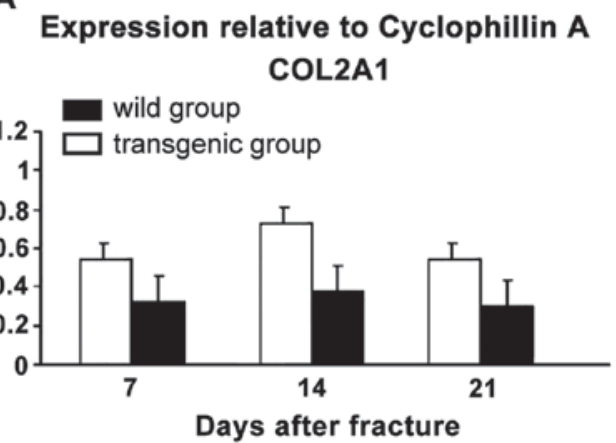

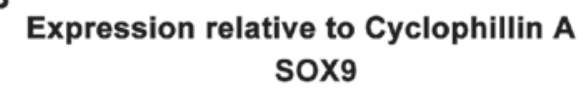

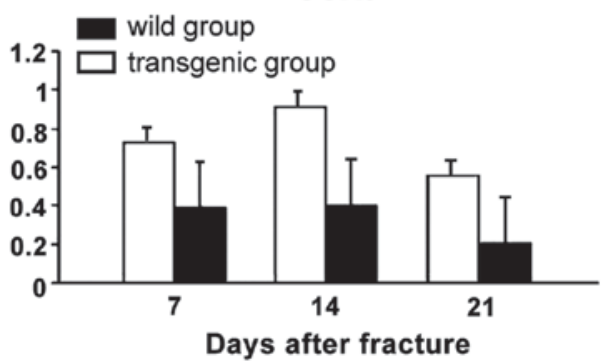

Figure 5. (A and B) Quantitative polymerase chain reaction.

mice at each time point after the fracture were markedly higher than that of the wild group (Fig. 5).

\section{Discussion}

NGF is a polypeptide protein that exists widely in various organs in animals. It is mainly produced by target tissue that is dominated by neurons in the neural crest. Neuron axon uptake is retrogradely transported to soma. NGF regulates the gene transcription of neuron cells in various ways, thus exerting a biological effect (8). In the 1990s, it was found that NGF may be produced in callus and immature tissues, which would induce nerve fibers growing into embryonic bone tissues and callus (9). Thus, NGF was capable of adjusting the growth and development of bone tissues and callus (9). Neuropeptide Y with sympathetic nerve component, peptidergic nerve fibre in intestinal vascular and alcitonin gene-related peptide with sensory nerve component, $\mathrm{p}$ substance and other peptidergic nerve fibers were present in callus (10). Various peptidergic nerve fibers existing in bone tissues are mainly distributed in metabolically active bone tissues, fracture sections, in particular. These fibers can significantly increase the expression of NGF and its receptor (11). Among the various osteoblast lineages cultured in vitro, there were not only NGF mRNA but also protein expression (12).

Fracture healing involves the stages of organization of hematoma, original callus formation and callus transformation and shaping. NGF is a bone resorption inhibitor that can increase bone reconstruction to avoid bone losses after fracture by reducing the excretion of proline and calcium (3). It can facilitate the process of callus chondrification and vascularization, and accelerate fracture healing through early cartilage mineralization of fracture ends (13). Previous findings have shown that, fractures treatment may be one of the diseases that are most appropriate for gene therapy (14). Grills et al found that callus formation, its stiffness and anti-bending strength were significantly increased in a local injection of NGF used to treat the fractured rib end of the mice (3). That result showed that a local injection of NGF facilitated the speed and efficiency of fracture healing, and played a significant role in each stage of fracture healing.

Through the high expression of endogenous NGF in transgenic mice, the present study has concluded that NGF mRNA expression levels in each callus formation phase were higher than those of the wild group on average. Fracture healing was completed in advance, residual cartilage decreased greatly, osteoclast formation increased and cartilage differentiation accelerated. The strain of NGF homozygous transgenic mice was relatively stable after 9 passages on average with rare gene mutation recovery. Additionally, compared with purely supplemented exogenous NGF, highly expressed NGF and its receptor could improve the NGF effect, avoid inadequate or excessive NGF doses of exogenous supplement, as well as allergic or other adverse reactions of the body (15). Findings of the current study showed that delayed fracture healing may be associated with an increase of the residual chondrocytes, chondrocyte differentiation barrier and slow cartilage matrix degradation. TRAP is an osteoclast characteristic enzyme. Degradation of 
the cartilage matrix is closely associated with the osteoclast absorption effect on the cartilage matrix (16). A large number of cartilage and mesenchymal tissues were evident in the middle and late stage of fracture healing because the COL2A1 expression of the chondrocyte marker gene in the wild group was significantly lower than that of the transgenic group (17). SOX9 gene, an important transcription factor for chondrocyte genesis and differentiation, expressed from the cartilage progenitor cell of mesenchyme, reached its expression peak at differentiated chondrocyte, and was significantly downregulated in hypertrophic chondrocytes (18). Previous studies were less involved in the relationship of NGF between chondrocytes in fracture healing (19). Current investigations on the promotion of NGF in fracture healing and newly-generated capillary are not comprehensive. Recently identified neurotrophins such as neuritin may constitute a downstream factor of neural activity and whether it would play a role in the promotion of NGF of the fracture healing process remains to be investigated (20).

In summary, NGF can promote cartilage differentiation, increase osteoclast formation and promote the healing of tibial fractures by increasing the levels of COL2A1 and SOX9 mRNA expression. The wide application of NGF in bone non-union, delayed healing and the prevention and treatment of osteoporosis greatly reduced morbidity and improved quality of life, providing greater understanding of the theory of the fracture healing mechanism.

\section{References}

1. Turner JE and Bosch JA: Closing the Border on a New Frontier: The problem with salivary nerve growth factor. Psychosom Med 78: 114-116, 2016.

2. Majuta LA, Longo G, Fealk MN, McCaffrey G and Mantyh PW: Orthopedic surgery and bone fracture pain are both significantly attenuated by sustained blockade of nerve growth factor. Pain 156: 157-165, 2015.

3. Grills BL, Schuijers JA and Ward AR: Topical application of nerve growth factor improves fracture healing in rats. J Orthop Res 15: 235-242, 1997.

4. Wang L, Zhou S, Liu B, Lei D, Zhao Y, Lu C and Tan A: Locally applied nerve growth factor enhances bone consolidation in a rabbit model of mandibular distraction osteogenesis. J Orthop Res 24: 2238-2245, 2006.

5. Zhuang YF and Li J: Serum EGF and NGF levels of patients with brain injury and limb fracture. Asian Pac J Trop Med 6: 383-386, 2013.

6. Rapp AE, Kroner J, Baur S, Schmid F, Walmsley A, Mottl H and Ignatius A: Analgesia via blockade of NGF/TrkA signaling does not influence fracture healing in mice. J Orthop Res 33: 1235-1241, 2015.
7. Kim SJ, Shin SJ, Choi NH and Cho SK: Arthroscopically assisted treatment of avulsion fractures of the posterior cruciate ligament from the tibia. J Bone Joint Surg Am 83-A: 698-708, 2001.

8. Liu Y, Zhao D, Wang W, Wang B, Liu Z, Zhang Y and Li Z: Nerve growth factor modulates bone morphogenetic protein expression in rabbit fracture. Zhonghua Yi Xue Za Zhi 94: 1825-1828, 2014 (In Chinese).

9. Frenkel SR, Guerra LA, Mitchell OG and Singh IJ: Nerve growth factor in skeletal tissues of the embryonic chick. Cell Tissue Res 260: 507-511, 1990.

10. Guo TZ, Wei T, Li WW, Li XQ, Clark JD and Kingery WS: Immobilization contributes to exaggerated neuropeptide signaling, inflammatory changes, and nociceptive sensitization after fracture in rats. J Pain 15: 1033-1045, 2014.

11. Ghilardi JR, Freeman KT, Jimenez-Andrade JM, Mantyh WG, Bloom AP, Bouhana KS, Trollinger D, Winkler J, Lee P and Andrews SW: Sustained blockade of neurotrophin receptors TrkA, TrkB and TrkC reduces non-malignant skeletal pain but not the maintenance of sensory and sympathetic nerve fibers. Bone 48: 389-398, 2011.

12. Yasui M, Shiraishi Y, Ozaki N, Hayashi K, Hori K, Ichiyanagi M and Sugiura Y: Nerve growth factor and associated nerve sprouting contribute to local mechanical hyperalgesia in a rat model of bone injury. Eur J Pain 16: 953-965, 2012.

13. Bei C, Lin Z, Yang Z, Zhao J, Su W, Sha K, Wei Q, Hua Q and Bo Z: Study on effect of NGF on fracture healing. Zhongguo Xiu Fu Chong Jian Wai Ke Za Zhi 23: 570-576, 2009 (In Chinese).

14. Ishihara A and Bertone AL: Cell-mediated and direct gene therapy for bone regeneration. Expert Opin Biol Ther 12: 411-423, 2012.

15. Mo Y, Yang Z, Zhao J, Su W, Sha K, Wei Q, Yang F, Hua Q and Ding X: Preliminary study on appropriate concentration gradient of nerve growth factor in promoting fracture healing. Zhongguo Xiu Fu Chong Jian Wai Ke Za Zhi 25: 575-581, 2011 (In Chinese).

16. Wang T, Wang Y, Menendez A, Fong C, Babey M, Tahimic CG, Cheng Z, Li A, Chang W and Bikle DD: Osteoblast-Specific Loss of IGF1R Signaling Results in Impaired Endochondral Bone Formation During Fracture Healing. J Bone Miner Res 30: 1572-1584, 2015.

17. Yang RC, Chen MH, Chen PY, Chen CY, Tsai SF, Cheng CK and Sun JS: A mutation of the Col2a1 gene (G1170S) alters the transgenic murine phenotype and cartilage matrix homeostasis. J Formos Med Assoc 113: 803-812, 2014.

18. Wang Z, Liang DC, Bai JY, Kang N, Feng JY and Yang ZQ: Overexpression of Sox9 gene by the lentiviral vector in rabbit bone marrow mesenchymal stem cells for promoting the repair of cartilage defect. Zhongguo Gu Shang 28: 433-440, 2015 (In Chinese).

19. Pecchi E, Priam S, Gosset M, Pigenet A, Sudre L, Laiguillon MC, Berenbaum $\mathrm{F}$ and Houard $\mathrm{X}$ : Induction of nerve growth factor expression and release by mechanical and inflammatory stimuli in chondrocytes: Possible involvement in osteoarthritis pain. Arthritis Res Ther 16: R16, 2014.

20. Wang X, Liu C, Xu F, Cui L, Tan S, Chen R, Yang L and Huang J: Effects of neuritin on the migration, senescence and proliferation of human bone marrow mesenchymal stem cells. Cell Mol Biol Lett 20: 466-474, 2015. 